\title{
The Quest for a Realistic Four-dimensional Cosmology
}

\author{
Ishwaree P Neupane \\ Department of Physics and Astronomy, University of Canterbury \\ Private Bag 4800, Christchurch, 8041 New Zealand \\ ishwaree.neupane@canterbury.ac.nz,ipn977@gmail.com
}

\begin{abstract}
:
The existence of a small and positive cosmological constant attributed to gravitational vacuum energy (or dark energy) in the present-day universe appears to be the most pressing obstacle as well as opportunity to significantly improving the models of four-dimensional cosmology from fundamental theories of gravity, including string theory and modern Kaluza-Klein theories. In seeking to resolve this problem, one naturally wonders if the real world can somehow be interpreted as an inflating de Sitter "brane" embedded in a five or even higher-dimensional space-time described by warped or non-factorizable geometry. In this scenario, the four-dimensional cosmological constant may well be determined in terms of two length scales: one is a scale associated with the size of extra dimensions and the other is a scale associated with the expansion rate of the universe, which is also related to the warping of extra spaces.
\end{abstract}

Physicists today are faced with a number of mysteries about the universe which to date have defied elegant and straightforward explanations. An illustrative example is the discovery, made nearly 12 years ago from observations of distant supernovae $[1,2]$ that the expansion of the universe is accelerating rather than slowing down. NASA's observations show that the kind of matter of which stars and galaxies are made forms less than 5\% of the universe's total matter-energy density, $\Omega_{\text {baryon }} \sim 0.05 \Omega_{\text {baryon }} \sim 0.05$ . Several other independent observations [3] indicate that about $22 \%$ of the total matte-energy density is in the form of non-baryonic cold dark matter (CDM) matter, $\Omega_{C D M} \sim 0.22 \Omega_{C D M} \sim 0.22$, which clump gravitationally but which have never been directly detected, and about $73 \%$ is in the form of mysterious dark energy, $\Omega_{v a c} \sim 0.73 \Omega_{v a c} \sim 0.73$. Physics behind the observed values of various cosmological parameters are still covered by a veil of mystery. For example, we do not know what dark energy and dark matter really are, although our universe is thought to be filled mostly with them. These scientific enigmas suggest we should look to new physics beyond the standard models of particle physics and cosmology.

It's very perplexing that astronomical observations favour a positive cosmological constant (or dark energy) in the present-day universe. Observational results further reveal that our universe experienced an inflationary epoch soon after its birth and that our universe now is mostly filled with dark energy and dark matter. But we do not know what the origin of gravitational vacuum energy driving inflation nor what dark energy and dark matter really are. In this sense, modern cosmology is facing three major mysteries: inflation, dark energy and dark matter. This all indicates toward the possibility that our well established theories of particle physics and general relativity are incomplete - or at least not fully understood. The success of the standard model of cosmology has itself raised several conceptual issues, such as, what makes up the dark side of the universe? Is gravity purely geometrical as Albert Einstein envisioned, or is there more to it (such as scalar fields, extra dimensions, supersymmetry)? What made our universe so big? This last question may be addressed by cosmic inflation, but again we do not know the physical origin of gravitationally repulsive vacuum energy driving inflation. Unprecedented experimental and theoretical efforts are currently being devoted to address these challenging questions in modern cosmology.

In the standard model of cosmology, first, we put in inflation - a period of exponential expansion that took place $10^{-35} \sec 10^{-35} \sec$ after the big-bang and that explains why the universe is smooth on the largest scales. Inflation can take a small homogeneous patch of space-time and turn it into something possibly larger than our currently observable universe, with all its essential features, such as, small density fluctuations discovered through the NASA's WMAP 
satellites [3]. These fluctuations are the seeds of modern galaxies. Second, we put in dark matter, which plays a key role in the formation of cosmic structure as well as holds the clusters and galaxies together. Third, we put in dark energy, which is used to balance the total energy budget of the universe and explain the accelerating expansion observed in the more recent history of our universe.

There are other ingredients that we often don't put in an effective four-dimensional theory but have reasons to suspect might be there. For example, we work with a Friedmann-Robertson- Walker universe described by three large dimensions of space and one time-like dimension even though we know that more (space-like) dimensions are possible, as implied by some fundamental theories of gravity, such as, superstring, supergravity and Kaluza-Klein theories.

The history of 20th century physics has been a struggle to find a way to unite general relativity and quantum mechanics. Detailed investigations have shown that the standard quantum field theory, as it is currently formulated, is incompatible with general relativity (at least in a four- dimensional spacetime), or vice versa, though both theories worked to wonderful effects in their own worlds. One of the ways in which physicists have tried to unify gravity with the other forces is to create theories that allow for many more spatial dimensions.

In the early 1980s, certain versions of ten- and eleven-dimensional supergraviy models, which are the low energy limits of 10D string theory and 11D M-theory, were realised as some useful theoretical tools in connecting quantum mechanics with general relativity. The major achievement in the mid1990s was a series of new understandings about the role of localized sources such as 'branes' and fluxes [4]. These ingredients in string/M-theory have helped us to learn several new ideas, including the localization of gravity on a brane embedded in a warped fivedimensional anti-de Sitter space [5], gravity/gauge theory dualities [7] and methods of constructing de Sitter vacua in string theory [6]. There is now a general consensus that modern string theory is a theory of one of the higher-dimensional objects called "branes" rather than just a theory of strings. Despite a few novelties, it is not straightforward to explain accelerating universes directly through a standard compactification of ten- or elevendimensional supergravity models. There is a 'no-go' theorem, due to Gibbons [8], Maldacena and Nunez [9] and many others, which basically assert that if we compactify any string-derived supergravity model on a smooth compact manifold, then an inflationary de Sitter solution is not realized as a background solution of 10D or 11D Einstein gravity. Since the universe appeared to be both past and future de Sitter (albeit with vastly differing vacuum energies) this would seem to be a problem.

The original no-go theorem assumes time independence of the internal space, and so one could look for time-dependent solutions. Following this intuition, the author and many others [10, 11] have constructed varieties of time-dependent compactifications which describe an accelerating universe. Nevertheless, in many examples studied in the literature, with maximally symmetric extra dimensions, we only obtain a transiently accelerating universe with time-dependent metric moduli or scalar fields. Furthermore, if the size of extra dimensions varies (with time), then there will be variations in Newton's constant and the fine structure constant. Strong experimental bounds on such variation place strong constraints on these models. To that plus some other reasons, one could look for an alternative scenario where the extra dimensions are warped but time-independent.

One of such proposals is a five-dimensional "warped" braneworld model pioneered by Randall and Sundrum [5]. In the socalled braneworld scenario our 4-dimensional universe is supposed to be a 4-dimensional timelike surface, or the world-volume of a 3-brane, in a higher dimensional spacetime. The RS braneworld proposal has actually raised the possibility that at least one of the extra dimensions postulated by string theory could be large enough to have experimental implications. An interesting implication of the braneworld model is that, when 
we go into the direction of the extra dimensions, all our scales will be stretched by a factor depending on the distance to our brane. In the RS single brane model, gravity is 'trapped' (on a brane) and extra dimensions can have infinite spatial extent, so called 'warped' geometry. For the original RS braneworld models to work, one needs a five-dimensional anti de Sitter space - a background geometry which is negatively curved. These also predict a zero cosmological constant on the brane, meaning that the usual $3+1$ spacetime is a ${ }^{\circ}$ at Minkowski spacetime. This last result is not supported by recent cosmological observations, which favour a de Sitter like universe. One thus finds interest to construct an explicit model that supports a positive cosmological constant on the brane or a 4D hypersurface.

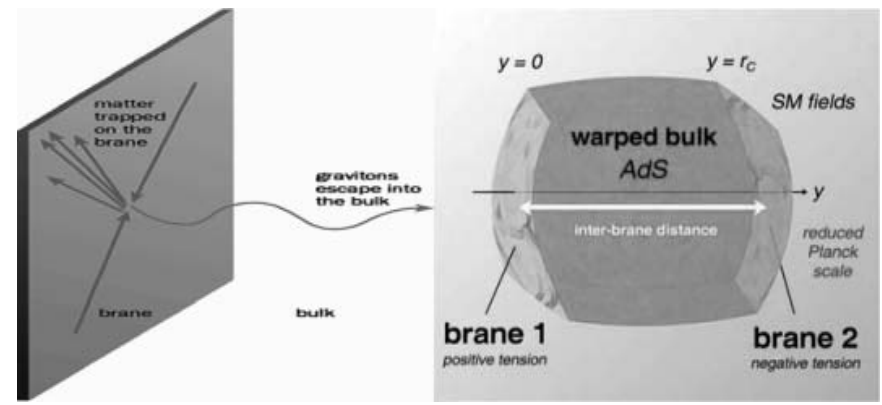

Figure 1: The picture on the left demonstrates the basic idea that gauge fields and non-gravitational particles could be trapped on a 3D brane but gravity can travel in the extra dimensions. The picture on the right shows a basic configuration of RS two-brane model where gravity resides on both branes, but its infuence on the second 'brane' at $y=r_{c} y=r_{c}$ is reduced by the warping of space between the two branes.

Whenweview ourobserveduniverseasa cosmological brane embedded in a higher dimensional warped space-time, the universe accelerates because of an effective 4D cosmological constant induced on the brane. This basic idea can be illustrated with a fivedimensional 'warped metric', maintaining the usual four-dimensional Poincare symmetry,

$$
{ }^{|y|}\left(\widehat{g}_{\mu v} d X^{\mu} d X^{v}+\rho^{2} d y\right) \text {, }
$$

where $\boldsymbol{X}^{\mu} \boldsymbol{X}^{\mu}$ are the usual space-time coordinates $(\mu=0,1,2,3), \rho$ is a length scale associated with the size of extra space and $e^{-K|y|} e^{-K|y|}$ is the warp factor as function of $y$. In the above $\mathbf{0} \leq \boldsymbol{y} \leq \boldsymbol{\pi}$ $\mathbf{0} \leq \boldsymbol{y} \leq \boldsymbol{\pi}$; there is some sort of 'brane' at the $y=0$ and $\boldsymbol{y}=\boldsymbol{\pi} \boldsymbol{y}=\boldsymbol{\pi}$ boundaries of a 5D bulk space-time. If $\widehat{\boldsymbol{g}}_{\mu v}=\boldsymbol{\eta}_{\mu v}, \widehat{\boldsymbol{g}}_{\mu v}=\boldsymbol{\eta}_{\mu \boldsymbol{v}}$, in which case the 'brane' is a flat four-dimensional Minkowski space, this metric describes a portion of 5D anti de Sitter space as in braneworld models.

Indeed, the metric (1) becomes an exact solution of 5D Einstein equations when

$$
\hat{g}_{\mu v} d X^{\mu} d X^{v}=-d t^{2}+a(t)^{2}\left[\frac{d r^{2}}{1-k r^{2}}+r^{2}\left(d \theta^{2}+\sin ^{2} \theta d \varphi^{2}\right)\right]
$$

and the scale factor of the universe $a(t)$ takes the form

$a(t)=\frac{1}{2} \exp \frac{K t}{\rho}+\frac{k \rho^{2}}{2 K^{2}} \exp \frac{-K t}{\rho}$,

where $K$ is an arbitrary constant (with inverse length dimension) and the spatial curvature constant $k=0 k=0, k=+1 k=+1$ and $k=-1 k=-1$, respectively, for flat, closed and open universes.

In the discussion below we move one of the branes to infinity, so that the second brane at $y=r_{c} y=r_{c}$ disappears from the scenario. For brevity we also assume that the 5D bulk cosmological term is absent and the standard model particles are confined or localised at $y=0$ rather than at $y=r_{c} y=r_{c}$. Now, to solve Einstein's equations on the $y=0$ boundary of space-time, we have to consider a 3-brane with brane tension $T 3$ and specify a boundary condition such that the warp factor is regular at $y=0$. The classical action describing this set up is

$S=\frac{M_{5}^{3}}{2} \int d^{5} x \sqrt{-g_{5}} R_{5}+\frac{M_{5}^{3}}{2} \int d^{4} x \sqrt{-g_{b}}\left(-T_{3}\right)$,

where $M_{5} M_{5}$ is the fundamental 5D Planck scale and $g_{b} g_{b}$ is the determinant of the metric $g_{a b} g_{a b}$ evaluated at the brane's position. Einstein's equations are explicitly solved at $y=0$ when

$$
T_{3}=\frac{12 K}{\rho} \text {. }
$$

Note that in this example the brane tension is induced by the curvature related to the expansion of $3+1$ space-time, which is determined in terms of two free parameters: the slope of warping $K$ and the $5 \mathrm{D}$ curvature radius $\otimes$. This result is quite different from the original RS models, where the warp factor is determined by a 5D bulk cosmological constant. More remarkably, in the case the warp factor is nonconstant (i.e. $|K|>0|K|>0$ ), the expansion of 20 
of higher-dimensional gravity models, including string theory, this suggestion may have startling implications.

First, the physical universe inflates naturally when the warping of extra space is nontrivial. This happens because the warping generates in the four-dimensional effective theory a cosmological constant-like term $\Lambda_{4} \Lambda_{4}$. This follows by substituting Eq. (1) into the action (4). Let us focus here on the $5 \mathrm{D}$ curvature term from which we derive the scale of gravitational interactions: $S_{e f f} \supset \frac{M_{5}^{3} \rho}{2} \int d^{4} x \sqrt{-\hat{g}_{4}} \int d y e^{-3 K|y|}\left(\hat{R}_{4}-\frac{12 K^{2}}{\rho^{2}}\right)$.

The second term inside the parenthesis behaves as an effective four-dimensional cosmological constant term, i.e. $\Lambda_{4}=6 \mathrm{~K}^{2} / \rho^{2} \Lambda_{4}=6 \mathrm{~K}^{2} / \rho^{2}$.

Second, as advertised above, the vacuum energy density is uniquely determined in terms of two length scales: one is a scale associated with the size of extra dimension, and the other is a scale associated with the expansion rate of the physical three spaces or the slope of the warp factor $K K$.

Third, from Eq. (6), we find that the relation between four- and five-dimensional effective Planck masses is given by

$M_{4}^{2}=M_{5}^{3} \rho \int_{-\infty}^{+\infty} d y e^{-3 K|y|} \approx \frac{2 M_{5}^{3} \rho}{3 K}$.

In the limit $K \rightarrow 0 K \rightarrow 0$, a new dimension of spacetime opens up, since $M_{4}^{2} \rightarrow \infty M_{4}^{2} \rightarrow \infty$ (or $G_{N} \rightarrow 0 G_{N} \rightarrow 0$ ), while with a nontrivial warp factor and a nonzero Hubble parameter) the 4D effective Newton's constant is finite. This result is generic and it holds when $D>5$.

The above discussion can be generalised by introducing a $5 \mathrm{D}$ cosmological constant term $\Lambda_{5} \Lambda_{5}$ : $S=\frac{M_{5}^{3}}{2} \int d^{5} x \sqrt{-g_{5}}\left(R_{5}-2 \Lambda_{5}\right)+\frac{M_{5}^{3}}{2} \int d^{4} x \sqrt{-g_{b}}\left(-T_{3}\right)$

and a more general 5D warped metric Ansatz, such as

$d s_{5}^{2}=e^{2 A(y)}\left(\hat{g}_{\alpha \beta} d X^{\alpha} d X^{\beta}+\rho^{2} d y\right)$.

With a nonzero $\Lambda_{5}, \Lambda_{5}$, the 5D Einstein equations

$G_{A B}=-\frac{T_{3}}{2} \frac{\sqrt{-g_{b}}}{\sqrt{-g_{5}}} g_{\mu \nu}^{b} \delta_{A}^{\mu} \delta_{B}^{v} \delta(\mathrm{y})-\Lambda_{5} g_{A B}$ are explicitly solved when the warp factor and 3-brane tension are given, respectively, by

$A(y)=\ln \left(24 K^{2}\right)-\ln \left(24 K^{2} e^{K|y|}+\Lambda \rho^{2} e^{-K|y|}\right)$

and

$T_{3}=\frac{24 K^{2}-\Lambda \rho^{2}}{2 \mu K}$.

These results are quite generic and they hold also when the number of dimensions $\mathrm{D}>5$.

The above example in five dimensions is sufficiently illustrative. Indeed, the internal consistency of string theory requires that the universe has six (or seven) more space-like dimensions if the universe is described by 10D string theory (or 11D M theory) than we are directly aware of. Therefore, from the viewpoint of string theory, it would be more relevant to generalize our discussion, by considering six more extra dimensions.

$d s_{10}^{2}=e^{2 A(y)} \hat{g}_{\mu v} d X^{\mu} d X^{v}+e^{\alpha A(z)} g_{m n}^{6}(y) d y^{m} d y^{n}$,

where $\rho$, as above, is the length scale associated with the size of extra spaces, $e^{A(y)} e^{A(y)}$ is the warp factor as function of one of the internal coordinates, $y$, and $\alpha$ is a constant.

From the viewpoint that space-time is an emergent phenomenon in the language of condensed matter physics, it seems quite plausible that some of the extra dimensions required by string theory are realized only when one probes a sufficiently high energy scale or small distances. In order to explain the fact that there are only three large spatial dimensions, one would have to find a way to deal with additional six dimensions, which is usually done by 'compactifying' the extra dimensions at very small scales.

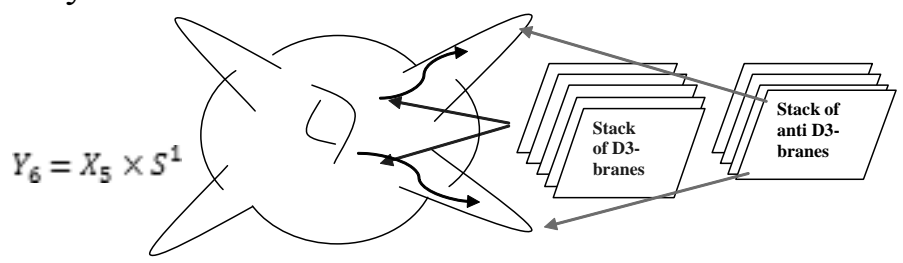

Fig 2. A stack of brane and antibranes embedded in a warped 6D geometry. Within string theory framework, dark matter is thought to be created when a pair of brane and antibrane annihilates. One of moduli scalar fields, corresponding to the physical separation between a D3 and antibrane D3-brane, also play the role of "inflaton". 
As an illustrative example, we will consider a sixdimensional warped conifold $Y 6$ which is a product of a radial dimension $R^{1} R^{1}$ and a compact $5 \mathrm{D}$ base space $X 5$, where $X 5$ is either a five-sphere or an Einstein-Sasaki space $T^{1,1}=\left(S^{2} \times S^{2}\right) \times S^{1}$ $T^{1,1}=\left(S^{2} \times S^{2}\right) \times S^{1}$ with metric

$d s_{X 5}^{2}=\frac{1}{9}\left(d \psi+\cos \theta_{1} d \varphi_{1}+\cos \theta_{2} d \varphi_{2}\right)^{2}+\frac{1}{6}\left(d \theta_{i}^{2}+\sin \theta_{i}^{2} d \varphi_{i}^{2}\right)$,

where $\left(\theta_{1}, \varphi_{1} \theta_{1}, \varphi_{1}\right)$ and $\theta_{2}, \varphi_{2} \theta_{2}, \varphi_{2}$ are coordinates on each $S^{2} S^{2}$ and $\psi$ is the coordinate of a $U(1)$ fiber. The full ten-dimensional warped metric space-time then takes the form

$d s_{10}^{2}=e^{2 A(y)} \hat{g}_{\mu \nu} d X^{\mu} d X^{v}+e^{\alpha A(y)} \rho^{2}\left(f_{1}(y) d y^{2}+\beta f_{2}(y) d s_{X 5}^{2}\right)$,

where $\alpha \alpha$ and $\beta$ are some constants. The functions $f 1(y)$ and $f 2(y)$ may be chosen as

$f_{1}(y)=\sinh ^{2}(y+\varepsilon), \quad f_{2}(y)=\cosh ^{2}(y+\varepsilon)$.

For these choice $f_{1}(y) f_{1}(y)$ and $f_{2}(y)$ $f_{2}(y)$ as above the radius of the $X_{5} X_{5}$ never vanishes. The $6 \mathrm{D}$ Ricci scalar curvature is $\quad R_{6}=20(1-\beta) /\left[\beta \cosh ^{2}(y+\varepsilon)\right]$ $R_{6}=20(1-\beta) /\left[\beta \cosh ^{2}(y+\varepsilon)\right]$, meaning that the internal space is Ricci flat only when $\beta=1$ $\beta=1$. The $6 \mathrm{D}$ metric is regular everywhere for any value of $\beta$. $\beta$
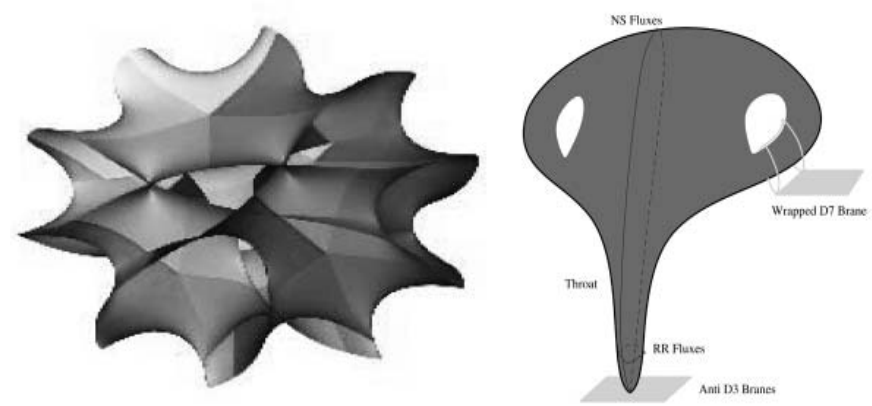

Figure 2: (left) A three-dimensional representation of a sixdimensional complex Calabi-Yau space. (right) A 6D conifold whose base is a $5 \mathrm{D}$ real manifold $T^{1,1}=\left(S^{2} \times S^{2}\right) \times S^{1}$ $T^{1,1}=\left(S^{2} \times S^{2}\right) \times S^{1}$.

To allow a de Sitter space solution in all three different cases: $R_{6}>0 R_{6}>0, R_{6}=0 R_{6}=0$, or $R_{6}<0 R_{6}<0$, we will consistently demand that $8 \beta=(2-\alpha)^{2} 8 \beta=(2-\alpha)^{2}$. In fact, the case $\alpha=2 \alpha=2$ must be treated separately. In the $\alpha \neq 2 \alpha \neq 2$ case, the $10 \mathrm{D}$ Einstein equations are explicitly solved when
$A(y)=\frac{1}{2-\alpha} \ln \frac{3 \mu^{2}(2-\alpha)^{2} \cosh ^{2}(y+\varepsilon)}{32}, \quad a(t)=\frac{1}{2} \exp \frac{K t}{\rho}+\frac{k \rho^{2}}{2 K^{2}} \exp \frac{-K t}{\rho}$.

This solution is interesting from at least two aspects; first, it is free from both space-like and time-like singularities, second it provides an explicit example of warped compactification on a four-dimensional de Sitter space without any background fluxes or supergravity sources.

Based on earlier braneworld no-go theorems [8, 9], it seemed almost impossible for these results to be true, but with what we presently understand [12], inflationary cosmology is possible for a wide class of metrics without introducing external fuxes or supergravity (brane) sources that violate certain positive energy conditions in the full $\mathrm{D}$ dimensional space-time. In [13], Gibbons and Hull constructed some other class of warped supergravity models that allow a de Sitter solution in the absence of fluxes. The solutions given in [13] are, however, singular at a point where the radius of $X_{5} X_{5}$ vanishes. The solution given above is regular everywhere. Interestingly enough, the Randall-Sundrum type mechanism, i.e. localization of gravity due to a nonsingular delta function source, is applicable to the present model.

Especially, in the $\alpha=2 \alpha=2$ case, we shall modify the 10D metric Ansatz as

$d s_{10}^{2}=e^{2 A(y)}\left[\hat{g}_{\mu \nu} d X^{\mu} d X^{v}+\rho^{2}\left(f_{1}(y) d y^{2}+f_{2}(y) d s_{\gamma 5}^{2}\right)\right]$.

This metric solves all of the $10 \mathrm{D}$ vacuum Einstein equations, when $f_{1}(y), f_{2}(y) \rightarrow f_{1}(y), f_{2}(y) \rightarrow$ const:

$d s_{10}^{2}=e^{-2 K|y|-K_{0}}\left[\hat{g}_{\mu v} d X^{\mu} d X^{v}+\rho^{2} e^{2 K_{0}}\left(2 K^{2} d z^{2}+d s_{X 5}^{2}\right)\right]$.

and the 4D scale factor takes the form

$a(t)=\frac{1}{2} \exp \frac{L t}{\rho}+\frac{k \rho^{2}}{2 L^{2}} \exp \frac{-L t}{\rho}, \quad L \equiv \sqrt{\frac{4}{3}} e^{-K_{0}}$.

This gives an explicit example of a non-singular warped de Sitter compactification. By introducing a new symbol $H$, such that $L / \rho \rightarrow \boldsymbol{H} L / \rho \rightarrow \boldsymbol{H}$, the $10 \mathrm{D}$ solution can be written as

$d s_{10}^{2}=e^{-2 K|y|-K_{0}}\left[\hat{g}_{\mu v} d X^{\mu} d X^{v}+\frac{4}{3 H^{2}}\left(2 K^{2} d z^{2}+d s_{X 5}^{2}\right)\right]$.

For this model to be phenomenologically viable one is required to satisfy $e^{-K_{0}} \ll 1 e^{-K_{0}} \ll 1$, see [12]. 
We conclude with a few remarks. The important ingredient in the above method of obtaining an inflationary de Sitter universe is the treatment of 'brane' as a four-dimensional physical spacetime and the consideration of a warped or non-factorizable background geometry in higher dimensions. Within this set-up and in dimensions $D \geq 5 D \geq 5$, inflationary cosmology has been shown to be possible with a wide class of metrics and with an arbitrary scalar curvature of the internal space. In our examples the 4D effective cosmological constant is determined by two parameters (at least to leading order): one

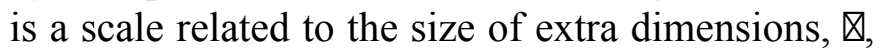
and the other is a scale associated with the curvature $L$ related to the expansion of the physical three spaces, that is, $\Lambda_{4}=6 L^{2} / \rho^{2} \Lambda_{4}=6 L^{2} / \rho^{2}$. The effective four-dimensional theory is thus Einstein's general relativity supplemented with a cosmological constant-like term. The approaches taken here are just some viable steps towards obtaining a scientific theory of four-dimensional accelerating cosmology from higher dimensional theories.

String theory as a theory of everything is sometimes criticized as unscientific because it is quite difficult to test by experiments or direct observations [14]. The controversy concerns two properties: First, like any other theory of quantum gravity, string theory seems to require extremely high energies to probe directly, higher by orders of magnitude than those that current experiments such as the Large Hadron Collider at CERN can reach. Second, string theory as it is currently developed and interpreted has a huge number of equally plausible solutions, called string vacua [6] and these vacua might be sufficiently diverse to explain almost any phenomena we might observe at lower energies or in a four-dimensional world. If these properties are true, string theory as a theory of everything or unified theory would have little or no predictive power for low energy particle physics experiments. Because the theory is so difficult to test, some theoretical physicists have asked if it can even be called a scientific theory (of quantum gravity). In the book The Trouble with Physics, Lee Smolin takes a complex debate on highly theoretical topics such as string landscape and ability of string theory to explain dark energy. But, it is difficult to imagine that a completely wrong theory could generate so many good ideas, including dualities between string theory and Yang-Mills gauge theory, microscopic descriptions of black holes and spacetime singularitie. Many tend to agree that string theorists' inability to empirically test their results may continue for sometimes to undermine their efforts.

Cosmology has been a rapidly developing, exciting area of science. In the coming decade one would hope to learn much more about inflation, dark energy and cosmic acceleration of the universe (attributed to a small positive cosmological constant) from the highly refined observations. Likewise we can hope to learn more about the true nature of dark matter from laboratory and new accelerator experiments that are underway as at the LHC. The dramatic developments in measurements of microwave background anisotropies that might within a few years allow a much more meaningful check of the very early universe cosmology, including inflationary paradigm. More and more data from precision cosmology might help to address questions about the early universe and the high energy frontiers. The years ahead will certainly bring even more twists, breakthroughs and surprises in cosmology research.

\section{References:}

1. G. Riess et al., Observational Evidence from Supernovae for an Accelerating Universe and a Cosmological Constant, Astron. J. 116 (1998) 1009 [arXiv:astro-ph/9805201];

2. S. Perlmutter et al., Measurements of Omega and Lambda from 42 High-Redshift Supernovae, Astrophys. J. 517 (1999) 565 [astro-ph/9812133].

3. D. N. Spergel et al. [WMAP Collaboration], First Year Wilkinson Microwave Anisotropy Probe (WMAP) Observations: Determination of Cosmological Parameters, Astrophysical Journal Suppl. 148 (2003) 175; D.N. Spergel et al. [WMAP Collaboration], Wilkinson Microwave Anisotropy Probe (WMAP) three year results: Implications for cosmology, Astrophysical Journal Suppl. $170 \quad$ (2007) 377 [arXiv:astro-ph/0603449]; WMAP Project Webpage [http://map.gsfc.nasa.gov].

4. J. Polchinski, Dirichlet-Branes and Ramond-Ramond Charges, Phys. Rev. Lett. 75, 4724 (1995) [arXiv:hepth/9510017].

5. L. Randall and R. Sundrum, A large mass hierarchy 
from a small extra dimension, Phys. Rev. Lett. 83 (1999) 3370; ibid, An alternative to compactification, Phys. Rev. Lett. 83, 4690 (1999) [arXiv:hepth/9906064].

6. S. Kachru, R. Kallosh, A. Linde and S. P. Trivedi, de Sitter vacua in string theory, Phys. Rev. D68 (2003) 046005 [arXiv:hep-th/0301240].

7. J. Maldacena, The large $N$ limit of superconformal field theories and supergravity, Adv. Theor. Math. Phys. 2, 231 (1998).

8. G. W. Gibbons, in Supersymmetry, Supergravity and Related Topics, edited by F. del Aguila, J. A. de Azcarraga, and L. E. Ibanz (World Scientific, 1985), pp. 123-146.

9. J. M. Maldacena and C. Nunez, Supergravity description of field theories on curved manifolds and a no go theorem, Int. J. Mod. Phys. A16, 822 (2001) [arXiv:hep-th/0007018].

10. P. K. Townsend and M. N. R. Wohlfarth, Accelerating cosmologies from compactification, Phys. Rev. Lett. 91 (2003) 061302 (2003) [arXiv:hep-th/0303097].
11. I. P. Neupane, Accelerating cosmologies from exponential potentials, Class. Quant. Grav. 21, 4383 (2004) [arXiv:hep-th/0311071]; I. P. Neupane and D. L. Wiltshire, Cosmic acceleration from $M$ theory on twisted spaces, Phys. Rev. D 72 (2005) 083509 [arXiv:hep-th/0504135]; I. P. Neupane, Accelerating universes from compactification on a warped conifold, Phys. Rev. Lett. 98, 061301 (2007) [arXiv:hepth/0609086].

12. I. P. Neupane, Extra dimensions, warped compactifications and cosmic acceleration, Phys. Lett. B683, 88 (2010) [arXiv:0903.4190]; Accelerating universe from warped extra dimensions, Class. Quant. Grav. 26 (2009) 195008 [arXiv:0905.2774].

13. G. W. Gibbons and C. M. Hull, de Sitter space from warped supergravity solutions, arXiv:hepth/0111072.

14. M. Chalmers, Stringscape, Physics World, September 2007 Issue, pp 35-47.

15. http://www.superstringtheory.com 